\title{
Programming relativity as the mathematics of perspective in a Planck unit Simulation Hypothesis
}

\author{
Malcolm Macleod \\ E-mail: maclem@platoscode.com
}

\begin{abstract}
The Simulation Hypothesis proposes that all of reality is in fact an artificial simulation, analogous to a computer simulation. Outlined here is a method for programming relativistic mass, space and time at the Planck level as applicable for use in Planck Universe-as-a-Simulation Hypothesis. For the virtual universe the model uses a 4-axis hyper-sphere that expands in incremental steps (the simulation clock-rate). Virtual particles that oscillate between an electric wave-state and a mass point-state are mapped within this hyper-sphere, the oscillation driven by this expansion. Particles are assigned an N-S axis which determines the direction in which they are pulled along by the expansion, thus an independent particle motion may be dispensed with. Only in the mass point-state do particles have fixed hyper-sphere co-ordinates. The rate of expansion translates to the speed of light and so in terms of the hyper-sphere co-ordinates all particles (and objects) travel at the speed of light, time (as the clock-rate) and velocity (as the rate of expansion) are therefore constant, however photons, as the means of information exchange, are restricted to lateral movement across the hyper-sphere thus giving the appearance of a 3-D space. Lorentz formulas are used to translate between this 3-D space and the hyper-sphere co-ordinates, relativity resembling the mathematics of perspective.
\end{abstract}

\section{Simulation Hypothesis}

The Simulation Hypothesis proposes that all of reality is an artificial simulation, analogous to a computer simulation [1]. The following discusses a method by which relativistic mass, space and time maybe be reproduced within a virtual (simulation) universe by embedding dual-state particles within a 4-axis hyper-sphere that expands in incremental steps [2]. In an article on a mathematical electron [3] was demonstrated how localized mass, length and time can be constructed from a particle oscillating between an electric-state (duration $=$ particle frequency) and a Planck-mass Planck-time point-state. This oscillation is a function of the expansion (the hyper-sphere acting as a pilot-wave). Likewise motion can be simulated by this expansion. By assigning an N-S axis to the particle, the direction in which the particle is 'pulled' by this expansion can be regulated, a transfer of momentum can then be simulated simply by changing the N-S axis orientation. In terms of the hyper-sphere co-ordinates the rate of expansion is the maximum velocity and so the simulation 'speed of light'. As all particles travel at this velocity, time and velocity are constants. Photons are a special case of particles without a mass point-state, as such they cannot travel in time and are restricted to lateral movement across the hyper-sphere. As all observable phenomena are measured using the electromagnetic spectrum, the radial hyper-sphere expansion can only be inferred, with photons giving the appearance of a relativistic 3-D space. The Lorentz formulas are used to translate between this 3 -D space and the hyper-sphere co-ordinate system, relativity then resembles the mathematics of perspective.

\section{Space-time}

Particle A is mapped onto a space-time graph (fig.1). A does not move in space $(v=0)$, but it does move in time.

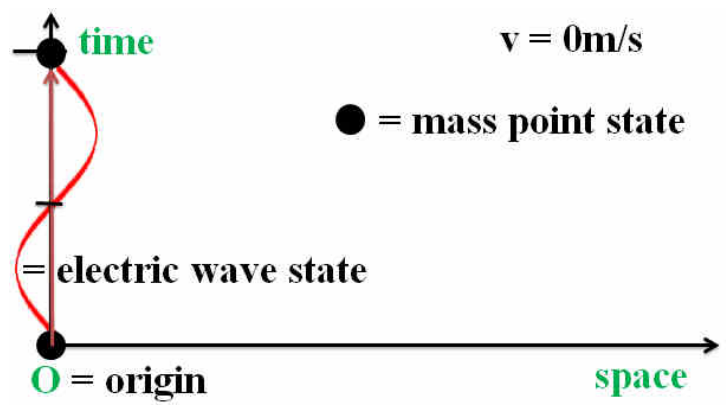

Fig. 1: particle $\mathrm{A}, \mathrm{v}=0$

Particle B, $v=0.866 c$ is added (fig.2). After 1s B will have traveled $0.866 \times 299792458=259620 \mathrm{~km}$ from A along the horizontal space axis.

Particles $\mathrm{A}$ and $\mathrm{B}$ both have a frequency $=6\left(5 t_{p}\right.$ in the electric wave-state then $1 t_{p}$ in the Planck mass $m_{P}$ point-state). As the A point-state occurs once every $6 t_{p}$, mass of $\mathrm{A} m_{A}=m_{P} / 6$, however the point-state of $\mathrm{B}$ occurs after $3 t_{p}$ along the $\mathrm{A}$ timeline and so $m_{B}=m_{P} / 3$ (fig.3).

As each step on the time axis involves $1 t_{p}$, there are 6 possible velocity solutions, this also means that $m_{B}$ can reach Planck mass $m_{P}$, but $\mathrm{B}\left(v=v_{\max }, m_{B}=m_{P} / 1\right.$, 


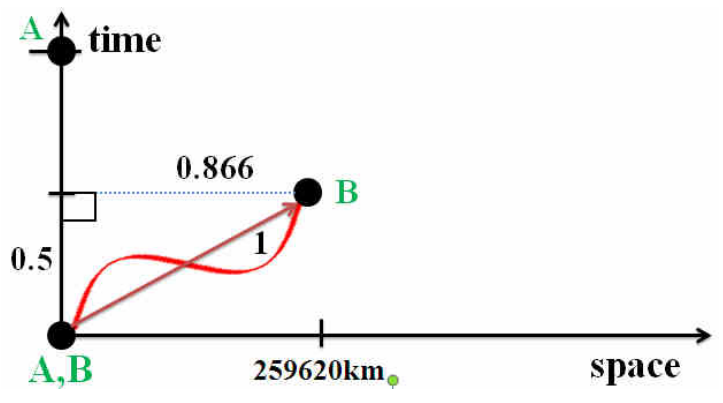

Fig. 2: particle $\mathrm{B}, \mathrm{v}=0.886 \mathrm{c}$

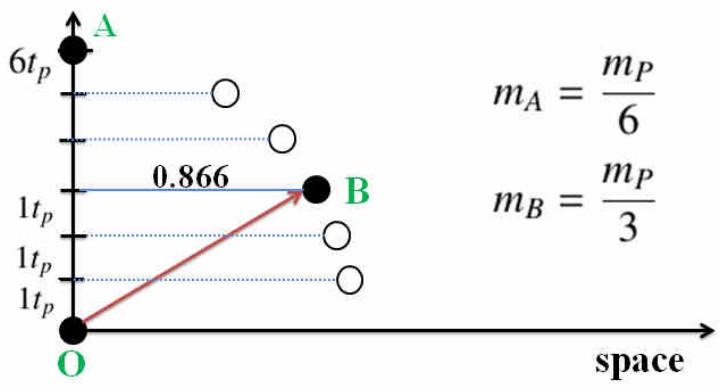

Fig. 3: particle B, relative mass

fig.4) can never attain the (horizontal axis) speed of light c.

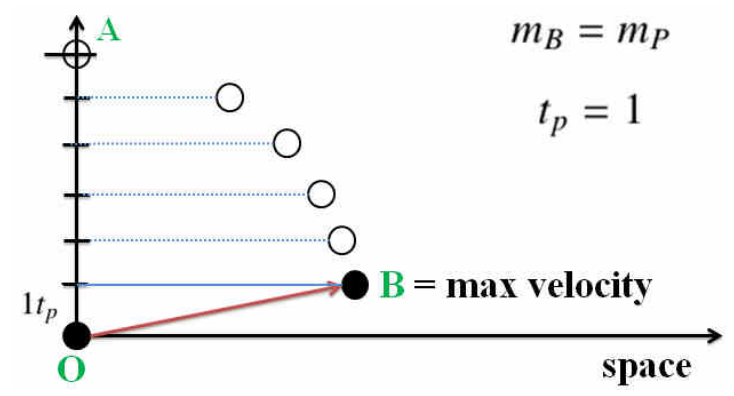

Fig. 4: particle B, maximum velocity

The vertical axis would be measured as $1 / \gamma$. For a particle that has only 6 divisions ( 6 steps from point to point), the maximum $\gamma=6$. To determine the maximum velocity that a particle can attain $(\mathrm{y}$-axis $=v / c)$ we simply calculate when that particle will have reached Planck mass, because from there it can go no faster. A small particle such as an electron has more divisions and so a higher $\gamma$ and so can go faster in 3-D space than a larger particle such as a proton with a smaller $\gamma$ (a smaller number of divisions).

$$
\begin{gathered}
\frac{1}{\gamma}=\sqrt{1-\frac{v^{2}}{c^{2}}} \\
\gamma_{\text {electron }}=m_{P} / m_{e}, \gamma_{\text {proton }}=m_{P} / m_{p}
\end{gathered}
$$

3 Hyper-sphere

3.1. Replacing the above with a 4 -axis co-ordinate system, to illustrate the concept are shown the $(h, x)$ axis with particles represented as semi-circles. Depicted is particle $\mathrm{B}$ at some arbitrary universe time $t$. B begins at origin $\mathrm{O}$ and is pulled along by the hyper-sphere pilot wave expansion (fig.5, 6, 7).

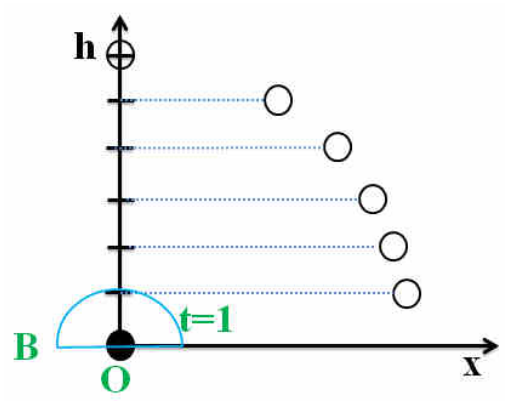

Fig. 5: $t=1$

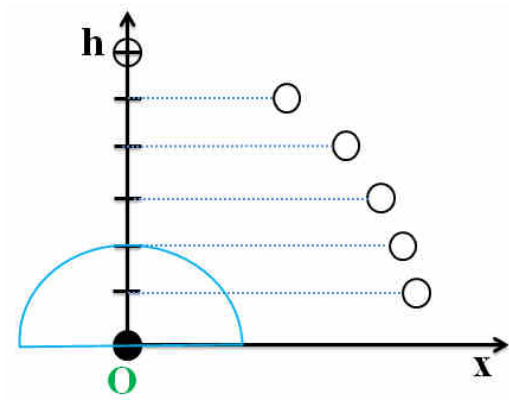

Fig. 6: $t=2$

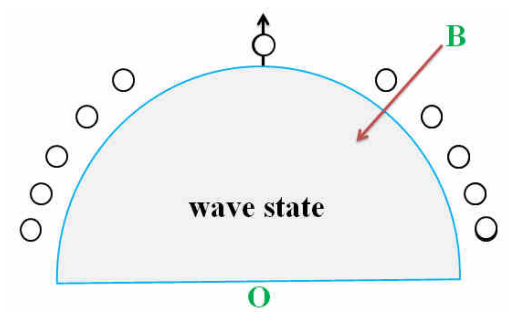

Fig. 7: $t=5$

At $t=6, \mathrm{~B}$ collapses into the mass point state and now has defined co-ordinates within the hypersphere (see fig.8) which then becomes the new origin $\mathrm{O}^{\prime}$, the above repeating ad infinitum $t=7,8, \ldots$ (fig.9, 10).

The process also repeats for A (fig.11). The hypersphere itself is then analogous to a particle presently in 


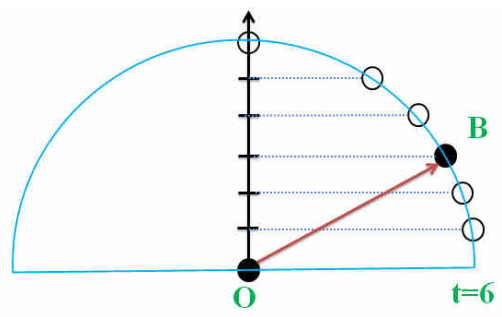

Fig. 8: $t=6$, point-state

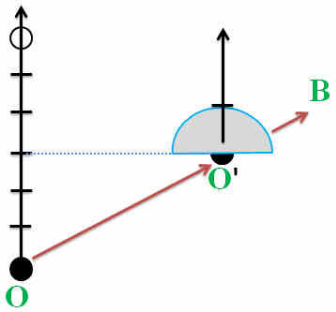

Fig. 9: $t=6+1$

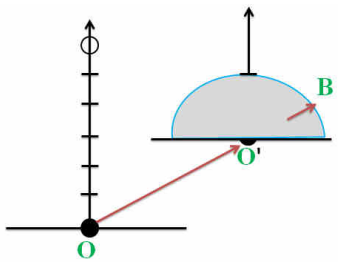

Fig. 10: $t=6+2$

the wave-state. Each particle origin $\mathrm{O}$ is thus repeating within the universe-as-a-particle hyper-sphere whose origin $\mathrm{O}$ was the big bang.

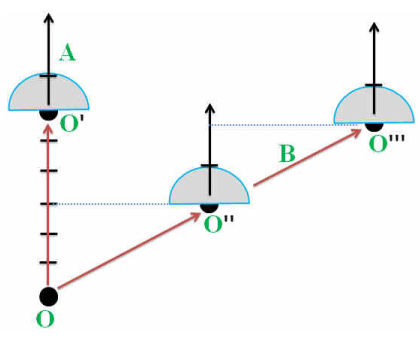

Fig. 11: Origin points; A, B

3.2. In the space-time diagram (fig.3) was depicted for $\mathrm{A}$; $v=0, m_{A}=m_{P} / 6$ and for $\mathrm{B} ; v=0.866 c, m_{B}=m_{P} / 3$. However in the $(h, x)$ graphs we find that as $\mathrm{A}$ and $\mathrm{B}$ have the same frequency, $f=6$, the lengths $\mathrm{OA}=\mathrm{OB}$ $=6$, this is because the hyper-sphere expands radially. As a consequence $\mathrm{B}$ can rightly claim that it is A whose velocity is at $v=0.866 c$ and for B velocity $v=0$ (fig.12).
Note that I am using relativistic mass instead of gammamass for purposes of illustrating the concept.

Both A and B are traveling at the speed of expansion (which translates to $c$ ) from the origin O. In the virtual coordinate system everything travels at, and only at, the speed of expansion as this is the origin of all motion, particles and planets do not have any inherent motion of their own, they are simply pulled along by this expansion.

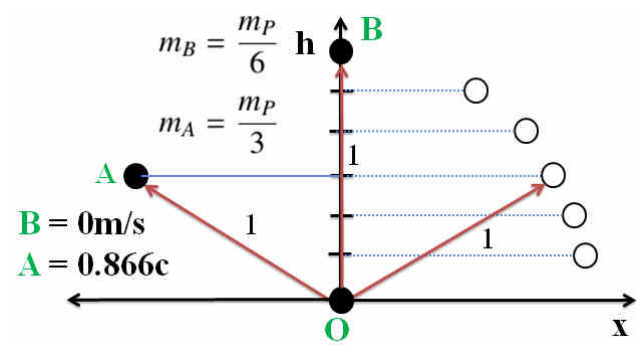

Fig. 12: relative mass $\mathrm{B}$ to $\mathrm{A}$

After 1 second both A and B will therefore have traveled the equivalent of $299792458 \mathrm{~m}$ in virtual co-ordinates from origin $\mathrm{O}$ (fig.13). Each of the 11 depicted solutions are equally valid as the radii are the same.

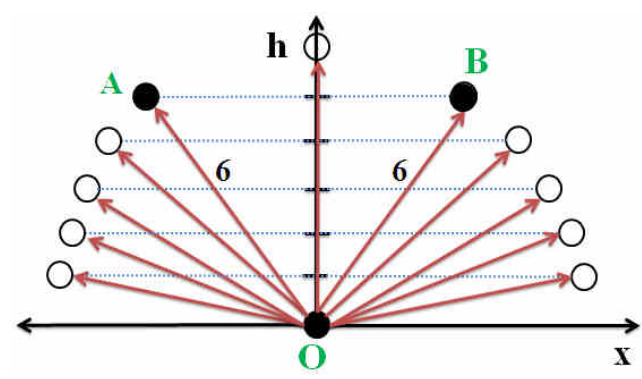

Fig. 13: radial expansion

3.3. Particles are assigned an N-S spin axis (fig.14). As the universe expands, it stretches particle A (position and motion of the wave-state are undefined). When $t=$ 6 , the wave-state collapses to the defined point-state, as determined by the $\mathrm{N}$. This means that of all the possible solutions, it is the particle N-S axis which determines where the point-state will actually occur, with the hypersphere acting as a pilot-wave.

Thus if we can change the N-S axis angle of B compared to $\mathrm{A}$, then as the universe expands the B wavestate will be stretched as with A. But the point of collapse will now reflect the new N-S axis angle. B does not need to have an independent motion; $\mathrm{B}$ is simply being dragged by the universe in a different direction as 


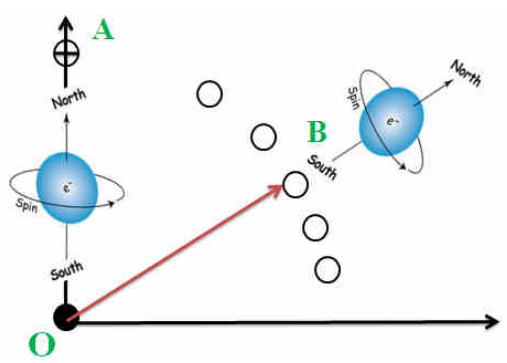

Fig. 14: N-S axis; $\mathrm{A} v=0, \mathrm{~B} v=0.886 \mathrm{c}$

the universe expands. We can thus simulate a transfer of physical momentum to B by simply changing the N-S axis. The radial universe expansion does the rest.

3.4. Information between particles is exchanged by photons. Photons do not have a mass point-state, only a wave-state, and so no means to travel the time-line axis (they are date-stamped). Instead they travel horizontally (and thus at the speed of light in 3-D space). The period required for particles to emit and to absorb photons is proportional to wavelength. In the following diagram (fig.15) A emits a photon. B travels towards A, as such it will take B less time to absorb that photon than if $\mathrm{B}$ was parallel to or moving away from $\mathrm{A}$. If the $\mathrm{x}$-axis length $x=v / c$, then the h-axis length $h=\sqrt{1^{2}-x^{2}}$ and the common relativistic Doppler equation can be written;

$$
v_{\text {observed }}=v_{\text {source }} \cdot \frac{\sqrt{1-\frac{v^{2}}{c^{2}}}}{1-\frac{v}{c}}=v_{\text {source }} \cdot \frac{h}{1-x}
$$

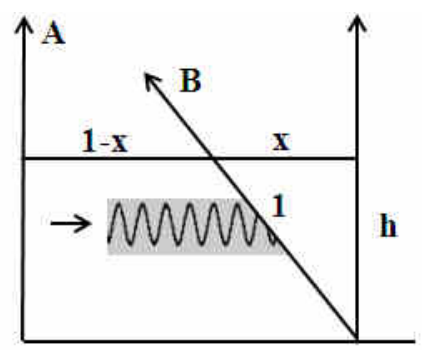

Fig. 15: Doppler shift

In the article on the virtual electron [3], the electron enters the mass point-state when the condition $(\mathrm{AL})^{3} / \mathrm{T}$ is reached where $\mathrm{T}=2 \pi$. If time $\mathrm{T}$ is not circular (rotation around an axis) but linear then this condition is not reached and the photon will remain in the wave-state (no mass point-state).

$E_{\text {wave }}=h v$ applies to both particle and photon wave states but $E_{\text {mass }}$ applies only to the particle mass pointstate. For each particle oscillation there is 1 Planckenergy wave-state followed by 1 Planck-mass point-state; and thus $E_{\text {wave }}=E_{\text {mass }}$, however as particle mass is the average frequency of occurrence of units of Planck mass then the formula $E=m c^{2}$ in the context of this model is misleading for $E=m c^{2}$ assumes particles have a constant property defined as mass [4].

3.5. Returning to our $\mathrm{ABC}$ particles, if photons (information) can only be exchanged along the horizontal axis which are the $(x, y, z)$ axis, ABC will only 'see' this horizontal information. Instead of virtual co-ordinates $\mathrm{OA}, \mathrm{OB}$ and $\mathrm{OC}$ and a constant time and velocity, the $(x, y, z)$ axis will be able to measure only the horizontal $\mathrm{AB}, \mathrm{BC}$ and $\mathrm{AC}$ (fig.16) as a $3-\mathrm{D}$ space.

There is no 'depth' perception, particle space will appear as a surface phenomena of the hyper-sphere but without a dimensional radius, the analogy being the entropy of a black-hole (akin to watching a 2-D radar display of aircraft above an airport).

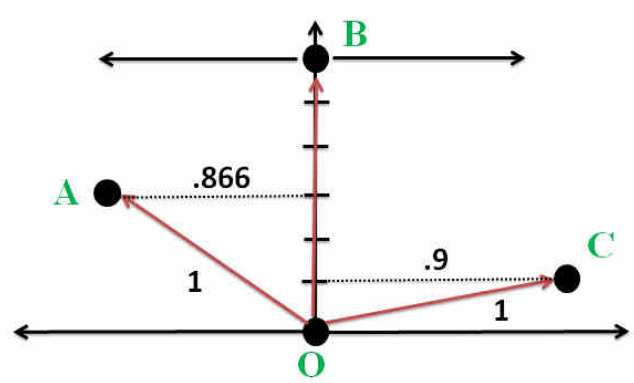

Fig. 16: 3-axis hyper-sphere surface

Furthermore time for $\mathrm{ABC}$ translates as motion, if there is no motion in the $(x, y, z)$ axis there will be no means to measure time, thus although the dimension of time for the 3-D space ABC world derives from simulation time (the clock-rate) and may equate to simulation time (as measured in units of Planck time), it is actually a measure of particle motion (a change of information states) and not the expansion.

This model uses a hyper-sphere expanding incrementally (the simulation clock-rate). If measured in units of Planck time, a 14.6 billion year old Planck universe (without including particles) will have similar parameters to the cosmic microwave background [2].

\section{References}

1. Nick Bostrom, Philosophical Quarterly 53 (211):243-255 (2003)

2. Macleod, Malcolm J., Programming a Planck blackhole Simulation Universe and the Cosmological constant

dx.doi.org/10.2139/ssrn.3333513 
3. Macleod, Malcolm J., Programming Planck units from a virtual electron; a Simulation Hypothesis

Eur. Phys. J. Plus (2018) 133: 278

4. Macleod, Malcolm J. "Plato's Cave; Source Code of the Gods", the philosophy and physics of a Virtual Universe, online edition

http://platoscode.com/ 